\title{
Bose-stimulated scattering off a cold atom trap
}

\author{
H. David Politzer* \\ California Institute of Technology, Pasadena, California 91125
}

(Received 29 August 1996)

\begin{abstract}
The angle and temperature dependence of the photon scattering rate for Bose-stimulated atom recoil transitions between occupied states is compared to diffraction and incoherent Rayleigh scattering near the BoseEinstein transition for an optically thin trap in the limit of large particle number $N$. Each of these three processes has a range of angles and temperatures for which it dominates over the others by a divergent factor as $N \rightarrow \infty$. [S1050-2947(97)01602-8]
\end{abstract}

PACS number(s): 03.75.Fi, 42.50.Gy, 05.30.Jp, 32.90.+a

\section{INTRODUCTION}

The quantum statistics of trapped atoms can drastically effect small-angle light scattering from a cold, degenerate cloud of gas. The scattering of an incident photon is accompanied by a transition between trap states of a recoiling atom. At small enough scattering angle or momentum transfer, the final atom state may very well already be occupied. For bosons there is an enhancement of the transition rate proportional to $n_{f}+1$, where $n_{f}$ is the number of bosons already in that final trap state. For fermions, transitions to occupied states are blocked; the analogous factor is $n_{f}-1$. (This Fock basis description is the natural one for the discussion of the phenomenon, particularly in the context of gaseous systems.) Considerable attention has been given to the consequent impact on resonant line shape [1]: the scattered photon energy is shifted up and down by the differences in trap-state energies, which are then averaged over the contributing processes in the thermal ensemble. However, the enhancement of the scattering rate itself and its angular dependence may also prove to be dramatic and interesting effects [2,3].

Reference [3] considered Born approximation scattering per unit volume for a plane wave of light in a uniform medium of bosonic atoms as a function of angle and temperature. As the temperature $T$ drops below the Bose-Einstein critical temperature $T_{c}$, as a consequence of this Bose enhancement, the scattering rate increases sharply for angles corresponding to momentum transfers $\delta$ such that the number of particles per $(\hbar / \delta)^{3}$ volume is large. While plane waves and infinite, uniform media are physically unattainable, they represent a reasonable approximation to a beam that is many wavelengths wide incident on the central region of a cloud that is many times larger still. This paper addresses another limit: when the whole trap is illuminated uniformly by the incident beam. In particular, I investigate the angle and $T$ dependence of the scattering rate for a plane wave incident on an isotropic, harmonic trap containing $N$ noninteracting atoms. Again, I consider only the Born approximation, i.e., a single scattering, which is appropriate to an optically thin trap. (This may be realized by a combination of low density and a sufficiently small basic cross section, e.g., achieved by sufficient detuning from resonance.) A

*Electronic address: politzer@theory.caltech.edu useful reference level is given by the isotropic Rayleigh scattering or fluorescence rate and a comparison with the even brighter coherent diffraction at yet smaller angles is made. In addition to the differential rates per solid angle, I also compare the total amounts of scattered light due to the various processes, integrated over all angles.

There are many possible strategies for doing this conceptually quite straightforward calculation. I have chosen to focus on the leading, large- $N$ behavior of each possible contributing subprocess for temperatures such that $T / T_{c} \sim O(1)$. In particular, while the trap ground state is treated explicitly, the other states are described semiclassically, e.g., with a continuous density of states and stationaryphase evaluations of WKB wave-function overlaps. This allows analytic evaluation (or at least estimates) of the various contributions to the total scattering. It proves quite enlightening because several subprocesses and regions of scattering angle scale differently with $N$ at fixed $T / T_{c}$. An alternative strategy of exact numerical evaluation would require considerable detail over a great range in $N$ to extract comparable information.

Laboratory detection of scattered light at very small angles has already been achieved in the context of cold atom traps. Dramatic images of condensation into a trap ground state have been obtained from coherently diffracted light [4]. Essentially, one diverges all small-angle light, blocks out the unscattered forward beam, and then focuses the remainder. Both diffraction and Bose-enhanced scattering have the properties that they are brighter (per unit solid angle) than the isotropic scattering and they do not contribute to heating the gas. Hence they both make possible nondestructive observation by allowing a greatly reduced illuminating intensity for a given detected signal strength. However, diffraction is confined to smaller and smaller angles with increasing trap size. Thus, for larger traps of the future, clean diffractive signals may be harder to extract. In contrast, while dimmer than diffraction at the smallest angles, the Bose-enhanced scattering contributes over a much larger range of angles a range that increases with $N$ - and for much of this region is considerably brighter than Rayleigh scattering.

In an infinite, uniform medium, diffraction cannot be addressed. It is of infinite intensity but relegated to zero angle. In contrast, the present analysis allows an explicit comparison of diffraction and Bose-stimulated scattering. This cal- 
culation also underscores the fact that the latter occurs only to the extent that the recoil from a photon can knock an atom from one occupied state to another and is proportional to the product of the two occupation numbers. As will become apparent, as $N \rightarrow \infty$ only a vanishing fraction of the total number of particles actively participate in the Bose enhancement. As a consequence, the total luminosity from Bose-stimulated scattering, integrated over angle, is significantly less than that from diffraction. On the other hand, diffraction reflects the overall particle density and its angular distribution scales inversely as the size of the system. In contrast, the Boseenhanced scattering depends on the momentum of particles in the occupied states. This momentum and, consequently, the typical scattering angle grow with increasing temperature.

This paper is organized as follows. Section I contains the thermal preliminaries. Section II contains the kinematics and the reference Rayleigh scattering. Section III discusses diffraction. Bose-stimulated scattering is evaluated in Sec. IV, first for transitions to and from the trap ground state and then for transitions between excited states; these are the only "new" formulas of this paper, but they make the most sense in the context of and in comparison with the other material. The qualitative lessons are summarized in Sec. V.

The division of the scattering physics into various subprocesses and the nomenclature used for them have been chosen to emphasize what is different in this situation and to facilitate the order of magnitude estimates of the various pieces and their dependences on $N$ and $T$. This gives rise to some terminology that is at variance with what is often used in optics, but the motivation is to enlighten rather than confuse. For example, "stimulated transition" refers traditionally to the light from an atom in response to the presence of other photons. In the present case, it is the motion of an atom in response to the presence of other atoms. Other variances arise because I focus on the dramatic angular dependence of the scattering rather than on the frequency spectra. Processes in which the scattered photon retains its energy are traditionally referred to as "elastic." In the present context, I call them diffractive because it is the physics of diffraction that determines their angular dependence. "Inelastic" processes are traditionally labeled variously depending on the source and magnitude of the energy exchange. The closest analog to the present situation is often called Brillouin scattering, with the Stokes and anti-Stokes processes referring to photon energy loss and gain. In the present case, atom transitions between two trap states make the same contribution to the photon angular distribution irrespective of the direction of the transition and the sign of the energy transfer. Hence they are treated here together. However, it is very useful to make a distinction between processes that involve atoms in the trap ground state from those that do not. This distinction is often not very relevant in other optics situations.

\section{THERMAL PRELIMINARIES}

It is convenient to use the natural units of the threedimensional isotropic harmonic oscillator. In particular, take

$$
m=\omega_{0}=\hbar=1,
$$

where $m$ is the particle mass and $\omega_{0}$ is the oscillator fundamental frequency. Also, measure temperature $T$ in energy units (i.e., the trap level spacing) so that Boltzmann's constant $k_{B}=1$. I will use Cartesian coordinates throughout the analysis. So the single-particle stationary states are labeled by a triplet of integers $\mathbf{m}=\left(m_{x}, m_{y}, m_{z}\right)$ and the energy of such a state can be taken to be $\varepsilon_{\mathbf{m}}=m_{x}+m_{y}+m_{z}$. (As is evident from the form of $\varepsilon_{\mathbf{m}}, \mathbf{m}$ is not a vector with respect to spatial rotations.)

Divide the total number of particles $N$ as $N=N_{0}+N_{e}$, where $N_{0}$ is the expected number of particles in the ground state (also written as $\left\langle n_{0}\right\rangle$ ) and $N_{e}$ is the expected number in excited trap states, i.e., the remainder. For $N \rightarrow \infty$, such a system has a critical temperature given by

$$
T_{c}=N^{1 / 3} \zeta(3)^{-1 / 3},
$$

where $\zeta(3) \simeq 1.202$ is the Riemann zeta function [5]. [In Eq. (1), and throughout, $T$ is measured in units of the trap level spacing; hence, to see a transition at fixed, finite, physical temperature, one must increase the trap size as $N \rightarrow \infty$.) For $T \leqslant T_{c}, N_{0}$ is a macroscopic fraction of $N$,

$$
N_{0}=N\left(1-\left(T / T_{c}\right)^{3}\right) .
$$

This can be deduced from the following evaluation of $N_{e}$. One begins by noting that the expected occupation of any state $\mathbf{m}$ is given by

$$
\left\langle n_{\mathbf{m}}\right\rangle=\frac{1}{e^{\left(\varepsilon_{\mathbf{m}}-\mu\right) / T}-1},
$$

where $\mu$ is the chemical potential. It follows that

$$
e^{-\mu / T}=1+\frac{1}{N_{0}}
$$

Hence, for all excited states we can take $\mu / T \simeq 0$ for $T \leqslant T_{c}$. Thus, for $T \leqslant T_{c}$,

$$
N_{e}=\int_{0}^{\infty} \frac{\frac{1}{2} m^{2} d m}{e^{m / T}-1}=T^{3} \zeta(3),
$$

which, by consistency, implies Eq. (2). The integral in Eq. (3) is obtained by approximating the sum over states $\mathbf{m}$, whose degeneracy is $\left(\varepsilon_{\mathbf{m}}+1\right)\left(\varepsilon_{\mathbf{m}}+2\right) / 2$ for large $N$ by an integral over $\varepsilon_{\mathbf{m}}$ (now called $m$ ). One can also deduce that imposing $N_{e}=N$ for $T \geqslant T_{c}$ implies

$$
\frac{\mu_{T \geqslant T_{c}}}{T} \simeq-\frac{18 \zeta(3)}{\pi^{2}} \frac{\left(T-T_{c}\right)}{T_{c}}+O\left(\left[\left(T-T_{c}\right) / T_{c}\right]^{2}\right) .
$$

The rate for a boson transition from a particular initial state $i$ to a particular final state $f$ is proportional to $n_{i}\left(n_{f}+1\right)$, where $n_{i}$ and $n_{f}$ are the actual occupation numbers. It is this product that then must be thermally averaged if the system as a whole is characterized by an equilibrium temperature. Hence, in principle, one needs to know the twolevel correlations $\left\langle n_{i} n_{f}\right\rangle$ as well as the occupation expectations $N_{i}=\left\langle n_{i}\right\rangle$. For the purposes of the present calculations, it suffices to use the replacement $\left\langle n_{i} n_{f}\right\rangle \simeq N_{i} N_{f}$ because the corrections to this form lead to changes in any final result 
that are down by $O(1 / N)$. (Here, "final result" refers to the result after the thermal average over all possible initial and final $\mathrm{N}$-atom states is performed.) The reasons are discussed at length elsewhere [6], but, briefly, the issues are as follows. For $i=f \neq 0$, the fractional fluctuations in the occupation of any substantially occupied state are large [i.e., $O(1)]$. However, for fixed $N$, the different $i$ 's fluctuate almost independently. So, for example, the variance associated with $N_{e}$ vanishes relative to $N_{e}$ as $N_{e} \rightarrow \infty$. Also, for $i \neq f$, the fixing of $N$ induces a correlation between occupations, but it is likewise negligible as $N \rightarrow \infty$. Finally, for $i=f=0$, fixing $N$ ensures that the fluctuations in $n_{0}$ are microscopic, even for an ideal gas with $T \leqslant T_{c}$.

\section{GENERAL KINEMATICS AND RAYLEIGH SCATTERING}

A photon of momentum $\mathbf{k}_{i}$ is incident on a trap. The goal is to estimate the differential cross section $d \sigma / d \Omega$ for scattering the photon to momentum $\mathbf{k}_{f}$ directed in the solid angle $\Omega$ (and also the total cross section $\sigma$ ). In particular, I integrate over the final photon frequency (or, equivalently, over $k_{f}$ ). In the natural units defined above, the unit of velocity is of order the rms zero-point velocity of an atom in the trap ground state. In these units, the speed of light $c$ is very large. If $\Delta \varepsilon$ is the change in the struck atom's energy in a particular collision and, therefore, minus the change in the photon's energy, then $k_{f}=k_{i}-\Delta \varepsilon / c$. Hence the scattering cross section $d \sigma / d^{3} \mathbf{k}_{f}$ is approximately proportional to $\delta\left(k_{f}-k_{i}\right)$. If the final goal is angular dependence, integrated over final photon energy, one can use the virtual equality of $k_{i}$ and $k_{f}$ to simplify the kinematics.

Let the momentum transfer be defined as $\boldsymbol{\delta}=\mathbf{k}_{f}-\mathbf{k}_{i}$. At small angles, using $k_{i} \simeq k_{f}, \delta$ is proportional to the scattering angle $\theta$,

and

$$
\theta \simeq \frac{\delta}{k_{i}},
$$

$$
d \Omega \simeq \delta d \delta d \varphi / k_{i}^{2},
$$

where $\varphi$ is the azimuthal angle. No information is lost by making the trivial simplification of ignoring the effects of photon polarization on angular dependence. (At issue is polarization in the scattering plane at large angles; if this were of interest, it could easily be accounted for correctly.) With this simplification, the photon angular dependence is $\varphi$ independent and totally determined by the magnitude of the momentum transfer to the atom $\delta$. Conversion to actual laboratory scattering angles involves knowing the incident photon momentum or frequency. However, the discussion of atomic trap physics is simplest and clearest in terms of $\delta$.

Born approximation scattering separates naturally into three types of processes: diffraction, Bose-stimulated scattering, and Rayleigh scattering. By "diffraction" I mean the collisions in which the struck atom remains in its original trap state. Hence the $N$-atom final state is the same as the $N$-atom initial state, irrespective of which atom is struck. Therefore, the amplitudes for photon scattering off each of the $N$ atoms must be added coherently and then the sum is squared to get the rate. When the struck atom changes state, even if unobserved, the rates for all the different possible processes are added (incoherently). As mentioned above, these rates are proportional to $n_{i}\left(n_{f}+1\right)$. The $n_{i} 1$ piece is what is traditionally identified as Rayleigh scattering. It is independent of the occupation of the final state. The $n_{i} n_{f}$ piece is what I mean by Bose-stimulated scattering. It is an analog for bosonic atoms of stimulated emission for photons.

In all cases, the key transition matrix element that determines the scattering connecting the trap state $i$ to $f$ is $\left\langle i\left|e^{i \boldsymbol{\delta} \cdot \mathbf{r}}\right| f\right\rangle$. This can be visualized in momentum space as the overlap of the initial momentum wave function shifted by the momentum transfer $\boldsymbol{\delta}$ with the final-state momentum wave function. However, many of the calculations are easiest to evaluate in position space.

The problem at hand is separable, e.g., in Cartesian coordinates. Hence the stationary-state wave functions $\langle\mathbf{r} \mid i\rangle$ are products of three one-dimensional harmonic-oscillator wave functions. Furthermore, the potential and the thermal distribution are isotropic. For convenience, we can choose one of our axes, e.g., $\hat{\mathbf{x}}$, to point along $\boldsymbol{\delta}$ and then

$$
\left\langle\mathbf{m}\left|e^{i \boldsymbol{\delta} \cdot \mathbf{r}}\right| \mathbf{m}^{\prime}\right\rangle=\left\langle m_{x}\left|e^{i \delta x}\right| m_{x}^{\prime}\right\rangle \delta_{m_{y}, m_{y}^{\prime} \delta_{m_{z}}, m_{z}^{\prime}}
$$

The one-dimensional wave functions of interest are the ground state

$$
\langle x \mid 0\rangle=\pi^{-1 / 4} e^{-x^{2} / 2}
$$

and the WKB wave functions for $m \gg 1$. For example, for $x>0$

$$
\begin{aligned}
\langle x \mid m\rangle \simeq & \left(\frac{\pi}{2}\right)^{-1 / 2}\left(2 m-x^{2}\right)^{-1 / 4} \\
& \times \begin{cases}\sin \left(\int_{x}^{a} \sqrt{2 m-x^{2}} d x+\frac{\pi}{4}\right) & \text { for } x<a \\
\frac{1}{2} \exp \left(-\int_{a}^{x} \sqrt{x^{2}-2 m} d x\right) & \text { for } x>a,\end{cases}
\end{aligned}
$$

where $a=\sqrt{2 m}$ is the classical turning point.

Rayleigh scattering serves as a convenient reference comparison for the other processes. The Rayleigh differential cross section is the total Rayleigh cross section for a single atom times the expression

$$
\sum_{i, f} N_{i}\left|\left\langle i\left|e^{i \boldsymbol{\delta} \cdot \mathbf{r}}\right| f\right\rangle\right|^{2}=N
$$

Hence Rayleigh scattering is independent of $T$ and $\boldsymbol{\delta}$ (i.e., isotropic) and proportional to $N$.

For the sake of simplifying all formulas and facilitating the comparisons of the various processes, I suppress the factor of the one-particle cross section and write all differential and total cross sections in units of that fundamental area. Hence I write 


$$
\begin{gathered}
\frac{d \sigma_{\text {Rayleigh }}}{d \Omega}=N, \\
\sigma_{\text {Rayleigh }}=4 \pi N .
\end{gathered}
$$

To reiterate, the displayed "cross sections" are not areas in the natural trap units; rather, they all have a single common factor, the atomic physics one-particle cross section, suppressed.

\section{DIFFRACTION}

The diffractive cross section is given by

$$
\frac{d \sigma_{\text {diffraction }}}{d \Omega}=\left|\sum_{i} N_{i}\left\langle i\left|e^{i \boldsymbol{\delta} \cdot \mathbf{r}}\right| i\right\rangle\right|^{2} .
$$

The $i$ th term in the sum is just the Fourier transform of the position space density of the $i$ th one-particle state, weighted by the occupation number. Hence Born approximation diffraction has a very classical interpretation, independent of the particle statistics: it is always given by the square of the Fourier transform of the density. (Of course, that density and its thermal behavior may themselves be very dependent on quantum statistics.)

For large $N$ and $T \sim O\left(T_{c}\right)$, it is appropriate to single out the ground state and treat the excited states semiclassically. In particular, the normalized position-space density for classical one-particle states of energy $m$ in a three-dimensional isotropic harmonic potential is

$$
\rho_{m}(\mathbf{r})=\frac{1}{\pi^{2} m^{2}} \sqrt{2 m-r^{2}}
$$

for $r^{2} \leqslant 2 m$ and 0 otherwise. In addition, the ground-state density is just

$$
\rho_{0}(\mathbf{r})=\pi^{-3 / 2} e^{-r^{2}}
$$

So the large- $N$ diffractive cross section is

$$
\begin{aligned}
\frac{d \sigma_{\text {diffraction }}}{d \Omega}= & \mid \int d^{3} \mathbf{r} e^{i \boldsymbol{\delta} \cdot \mathbf{r}}\left[N_{0} \rho_{0}(\mathbf{r})\right. \\
& \left.+\int_{0}^{\infty} \frac{\frac{1}{2} m^{2} d m}{e^{(m-\mu) / T}-1} \rho_{m}(\mathbf{r})\right]\left.\right|^{2} \\
= & \left|N_{0} e^{-\delta^{2} / 4}+\frac{4 T}{\delta^{4}} \int_{0}^{\infty} \frac{d z}{z^{3}} e^{-1 / z} e^{\delta^{2} \mu z / 2}\right|^{2} \\
& =\left|N_{0} e^{-\delta^{2} / 4}+\frac{4 T}{\delta^{4}}\right|^{2} \text { for } \mu=0 .
\end{aligned}
$$

[The $z$ integral in Eq. (6) is an approximation to an exact infinite sum that arises in the evaluation of the preceding line; the integral representation is valid for $\delta^{2} T \gg 1$.] Note that

$$
\frac{4 T}{\delta^{4}}=\left(\frac{2}{\delta^{2} T}\right)^{2} \frac{N_{e}}{\zeta(3)} .
$$

Also, if the characteristic linear size of the diffuse cloud of atoms in excited trap states at temperature $T$ is called $L$, then $L^{2} \sim O(T)$. The corresponding, characteristic momentum transfer supported by diffraction from such a cloud, $\delta_{L}$, would be $O(1 / L)$; so $\delta_{L}^{2} T \sim O(1)$. Thus the two terms in $d \sigma_{\text {diffraction }} / d \Omega$ have a clear interpretation. The diffraction cross section is nominally $O\left(N^{2}\right)$; however, the $N_{0}^{2}$ part has support only for $\delta \lesssim O(1)$, i.e., the inverse of the spatial extent of the ground state. The excited-state part has power falloff in $\delta$ and is only $O\left(N_{e}^{2}\right)$ or larger for $\delta \lesssim O\left(T^{-1 / 2}\right) \sim O\left(N^{-1 / 6}\right)$, where the latter estimate applies for $T \sim O\left(T_{c}\right)$. Of course, in the square of the amplitudes, there is also a cross term between the two.

[For completeness, I note that for $\mu<0$ the $z$ integral in Eq. (6) can be expressed as a sum of two "modified Bessel functions of the second kind" of ranks 0 and 1 and argument $\delta \sqrt{-2 \mu}$. However, the qualitative and asymptotic behavior is easiest to extract from the integral representation itself.]

To integrate over angles, one must recall Eq. (4) for $d \Omega$. The excited-state part of the diffractive cross section appears to be singular as $\delta \rightarrow 0$. However, that is a reflection of the inappropriateness of representing the discrete states as a continuum when considering very small shifts in momentum. If one simply excludes the vanishingly small forward cone as $N \rightarrow \infty$ in which the diffuse cloud diffraction contributes, the remaining diffraction is dominantly off the condensate:

$$
\sigma_{\text {diffraction }} \simeq \frac{2 \pi}{k_{i}^{2}} N_{0}^{2}
$$

Alternatively, one can estimate where the discreteness of the spectrum would cut off the integral. For excited states with energies of $O(T)$, the difference in the rms momentum from one level to the next is $O\left(T^{-1 / 2}\right)$. Using that as an estimate for the smallest meaningful $\delta$ in integrating Eq. (7), one learns that the integrated cross section due to excited states alone is $O\left(N_{e}^{5 / 3} / k_{i}^{2}\right)$, which does not compete with Eq. (8) for $T<T_{c}$ as $N \rightarrow \infty$.

The explicit appearance of the incident photon momentum $k_{i}$ is inevitable here (and also below) when considering total cross sections. While the Rayleigh differential cross section is genuinely isotropic, diffraction and Bosestimulated scattering are only significant at small angles. Within those ranges they can be much larger than the Rayleigh rate, as indicated by the formulas. However, the extent of those ranges is governed by the ratio of the acceptable struck-atom momentum transfer (which depends on trap geometry and temperature) to the incident photon momentum, which is typically much larger.

\section{BOSE-STIMULATED SCATTERING}

The Bose-stimulated rate has two sources: scattering atoms into and out of the condensate and scattering atoms between excited trap states. For each of these classes of processes, one first determines the square of the matrix element 
of $e^{i \boldsymbol{\delta} \cdot \mathbf{r}}$ between initial and final states. Again, it is convenient to use $\delta$ to define one of the Cartesian coordinate directions, e.g., $\boldsymbol{\delta}=\delta \hat{\mathbf{x}}$. Then the relevant overlap is an integral over one-dimensional oscillator wave functions. Also, only states that share the same $\hat{\mathbf{y}}$ and $\hat{\mathbf{z}}$ quantum numbers contribute, as indicated in Eq. (5). So the thermal sum over occupations of possible states involves first a projection onto the contributing states and then a sum over the thus-projected occupations weighted by the nontrivial one-dimensional matrix element squared.

\section{A. In and out of the condensate}

With the choice $\hat{\mathbf{x}}=\boldsymbol{\delta} / \delta$, only states labeled by $\mathbf{m}=(m, 0,0)$ have nonzero matrix elements of $e^{i \boldsymbol{\delta} \cdot \mathbf{r}}$ with the ground state. The one-dimensional overlap of relevance for transitions between the trap ground and excited states is

$$
F(m, \delta) \equiv\left|\left\langle 0\left|e^{i \delta x}\right| m\right\rangle\right|^{2} .
$$

For large $N$ and $T \sim O\left(T_{c}\right)$, only $m \gg 1$ is of interest. $F$ is sharply peaked around $\delta \approx \sqrt{2 m}$, the maximum classical momentum of a one-dimensional harmonic oscillator of energy $m$. Its width in $\delta$ is $O(1)$, which is vanishingly small on the scale of the typical value of $\sqrt{m}$. Hence it suffices to represent $F$ as a $\delta$ function whose normalization can be deduced by integrating $F$ over $\delta$. The result is

$$
F(m, \delta) \simeq \delta\left(m-\frac{\delta^{2}}{2}\right)
$$

This behavior has a simple classical interpretation. To bring an energetic particle in a three-dimensional harmonic potential to rest with a single impulse and have it remain at rest, the following must be true: the energetic orbit must pass through the origin, the impulse must be applied when the particle passes through the origin, and the impulse must be precisely that required to bring the particle to rest.

The Bose-stimulated differential cross section is obtained by summing over $m$ the equal contributions of transitions $0 \rightarrow m$ and $m \rightarrow 0$ :

$$
\begin{aligned}
\frac{d \sigma_{\text {Bose }}^{0, m}}{d \Omega} & =2 N_{0} \int_{0}^{\infty} \frac{d m}{e^{m / T}-1} F(m, \delta) \\
& =\frac{2 N_{0}}{e^{\delta^{2} /(2 T)}-1} \approx \begin{cases}2 N_{0} e^{-\frac{\delta^{2}}{2 T}} \text { for } \delta^{2} \gtrsim 2 T \\
4 N_{0} \frac{T}{\delta^{2}} & \text { for } \delta^{2}<2 T .\end{cases}
\end{aligned}
$$

For $\delta \sim O(1)$ this is $O\left(N_{0} N_{e}^{1 / 3}\right)$, while the diffractive cross section is $O\left(N_{0}^{2}\right)$; however, the latter then falls off exponentially with $\delta^{2}$, while the $0 \leftrightarrow m$ stimulated rate falls only as $1 / \delta^{2}$. Note also that this stimulated rate is much larger than the Rayleigh rate as long as $\delta<O\left(T^{1 / 2}\right)$. When $\delta^{2}>T$, the $0 \leftrightarrow m$ stimulated rate falls off exponentially with $\delta^{2} / 2 T$ because of the falloff in thermal occupation of appropriately high momentum states.

This analysis is again inadequate for very small $\delta$, where the discreteness of the spectrum is relevant. However, we can integrate down to $\delta \sim O(1)$ for which these approximations are sufficient and estimate

$$
\sigma_{\text {Bose }}^{0, m}(\delta \gtrsim 1) \simeq \frac{4 \pi N_{0} T}{k_{i}^{2}} \ln (2 T) .
$$

\section{B. Between excited states}

The excited states $\mathbf{m}$ and $\mathbf{m}^{\prime}$ must be related by $\mathbf{m}=\left(m, m_{y}, m_{z}\right)$ and $\mathbf{m}^{\prime}=\left(m^{\prime}, m_{y}, m_{z}\right)$ to get a nonzero overlap with $e^{i \boldsymbol{\delta} \cdot \mathbf{r}}$. The relevant one-dimensional matrix element is

$$
G\left(m, m^{\prime}, \delta\right) \equiv\left|\left\langle m\left|e^{i \delta x}\right| m^{\prime}\right\rangle\right|^{2}
$$

This is to be summed against the thermal occupations projected onto the one dimension:

$$
\begin{aligned}
\mathcal{P}\left(m, m^{\prime}, T, \mu\right) \equiv & \sum_{m_{y}, m_{z}}\left[\left(e^{\left(m+m_{y}+m_{z}-\mu\right) / T}-1\right)\right. \\
& \left.\times\left(e^{\left(m^{\prime}+m_{y}+m_{z}-\mu\right) / T}-1\right)\right]^{-1} \\
= & T^{2} P\left(\frac{m-\mu}{T}, \frac{m^{\prime}-\mu}{T}\right),
\end{aligned}
$$

where

$$
P(a, b) \equiv \int_{0}^{\infty} \frac{z d z}{\left(e^{z+a}-1\right)\left(e^{z+b}-1\right)} .
$$

Thus, in terms of the functions so defined,

$$
\frac{d \sigma_{\mathrm{Bose}}^{m, m^{\prime}}}{d \Omega}=\int d m d m^{\prime} G\left(m, m^{\prime}, \delta\right) \mathcal{P}\left(m, m^{\prime}, T, \mu\right) .
$$

$P(a, b)$ can be expressed in closed form in terms of exponentials, logarithms, and dilogarithms. Again, though, its qualitative and limiting behavior is easiest to see from the defining integral, Eq. (9). $P(a, b)$ is symmetric in $a$ and $b$ and it is $O(1)$ when both $a$ and $b$ are themselves $O(1)$. If $b \leqslant a \ll 1$, it is $O(\ln (1 / a))$. In addition, it is $O(\exp (-a-b))$ when $a, b>1$. [Recall, also, that $\mu / T$ is 0 for $T \leqslant T_{c}$ and $O\left(\left(T-T_{c}\right) / T_{c}\right)$ for $\left.T \gtrsim T_{c}.\right]$

$G\left(m, m^{\prime}, \delta\right)$ can be evaluated by stationary phase using the WKB wave functions. For each $m, m^{\prime}$, and $\delta$ (all three positive), there is a single position-space point of stationary phase in the overlap integral, corresponding to the unique classical position where an impulse of magnitude $\delta$ can change a particle of energy $m$ to one of energy $m^{\prime}$. $G\left(m, m^{\prime}, \delta\right)$ is symmetric in $m, m^{\prime}$. The following simple form is for $m \geqslant m^{\prime}$ :

$$
\begin{aligned}
& G\left(m, m^{\prime}, \delta\right) \\
& =\left\{\begin{array}{l}
\frac{1}{2 \pi}\left[2 m^{\prime} \delta^{2}-\left(m-m^{\prime}-\frac{\delta^{2}}{2}\right)^{2}\right]^{-1 / 2} \\
0 \quad \text { for } 2 m^{\prime} \delta^{2}<\left(m-m^{\prime}-\frac{\delta^{2}}{2}\right)^{2}
\end{array}\right.
\end{aligned}
$$

Unlike $F(m, \delta)$, the support of $G\left(m, m^{\prime}, \delta\right)$ for $m, m^{\prime} \gg 1$ extends over a non-negligible region in $\delta$ relative to the typi- 
cal thermal values of $m$ and $m^{\prime} . G\left(m, m^{\prime}, \delta\right)$ has an integrable square-root singularity on its boundary in the $m-m^{\prime}$ plane. That singularity occurs when the point of stationary phase is the origin in position space. The stationary-phase evaluation takes account not only of how likely it is to find the particle at the point of stationary phase, but also how rapidly the phase begins to change as one goes away from that point. It is this latter aspect that is optimized by applying the impulse when the particle is at the origin, and that is where the maxima of $G\left(m, m^{\prime}, \delta\right)$ occur.

Combining these factors, one finds, e.g., for $T \leqslant T_{c}$,

$$
\begin{aligned}
\frac{d \sigma_{\text {Bose }}^{m, m^{\prime}}}{d \Omega}= & 2 \int_{\delta^{2} / 8}^{\infty} d m \int_{(\sqrt{m}-\delta / \sqrt{2})^{2}}^{m} d m^{\prime} G\left(m, m^{\prime}, \delta\right) \\
& \times \mathcal{P}\left(m, m^{\prime}, T, 0\right) \\
= & \frac{T^{3}}{\pi} \int_{a / 4}^{\infty} d x \int_{x+a-2 \sqrt{a x}}^{x} d y \int_{0}^{\infty} z d z\left\{\left(e^{z+x}-1\right)^{-1}\right. \\
& \times\left(e^{z+y}-1\right)^{-1}(y-x-a+2 \sqrt{a x})^{-1 / 2} \\
& \left.\times(x+a+2 \sqrt{a x}-y)^{-1 / 2}\right\}
\end{aligned}
$$

where

$$
a=\frac{\delta^{2}}{2 T} .
$$

Hence $d \sigma_{\text {Bose }}^{m, m^{\prime}} / d \Omega$ is $T^{3}$ (remember that $T \simeq N_{e}^{1 / 3}$ ) times a function $f$ of the ratio $a$ defined above. When $a \sim O(1)$, $f(a)$ is $O(1)$. When $a \gg 1, f(a)$ is $O(\exp (-a / 4))$. In addition, when $a \ll 1, f(a)$ is still $O(1)$. To ascertain this last feature, note that the range of integrated $y$ vanishes as $a \rightarrow 0 ; y$ can be estimated as $x$ in the $z$ integral, yielding a $\ln (1 / x)$ integrable singularity upon integrating $z$, while the singularities in the $y$ integral itself are also integrable, yielding approximately $\pi / 2$. Hence the apparent divergence of the $z$ integral (as $x, y \rightarrow 0$ ) is not realized, even for $a \rightarrow 0$. A summary of these estimates can be represented as

$$
\frac{d \sigma_{\mathrm{Bose}}^{m, m^{\prime}}}{d \Omega} \sim O\left(N_{e} e^{-\delta^{2} / 8 T}\right)
$$

for all $\delta$.

This is the crudest of the explicit determinations of the coherent processes [simply because Eq. (10) is the furthest from a closed form in terms of elementary functions]. However, it is also the only coherent process whose differential rate never exceeds the Rayleigh rate by a divergent factor as $N \rightarrow \infty$. So there is no range of angle or temperature for which it is the overwhelmingly dominant process. A numerical determination of $f(a)$ is certainly feasible, but the detailed result would shed no light on the present, general discussion. It would be needed, though, in the context of a particular trap potential for a particular experiment if one wanted to account for all the light at each angle to better than a factor of 2 or 3.

From the estimated behaviors given above, one can estimate the $m, m^{\prime}$ contribution to the cross section:

$$
\sigma_{\mathrm{Bose}}^{m, m^{\prime}} \sim O\left(\frac{N_{e}^{4 / 3}}{k_{i}^{2}}\right),
$$

which is down by a factor of $\ln (T)$ relative to $\sigma_{\text {Bose }}^{0, m}$ at least when $N_{0}$ is comparable to $N_{e}$, because the differential $m \leftrightarrow m^{\prime}$ cross section does not grow like $T / \delta^{2}$ as $\delta^{2}$ decreases below $T$.

\section{SUMMARY AND CONCLUSIONS}

I have chosen units and notation to emphasize the angle, temperature, and number dependence of light scattering off an optically thin cold atom trap. The angle is best represented in a general discussion by the momentum transfer $\boldsymbol{\delta}$. I have focused on temperatures $T$ near the Bose-Einstein condensation temperature $T_{c}$. In addition, I have used semiclassical analytic techniques to evaluate the leading large- $N$ (particle number) behavior of each type of contributing process.

Rayleigh scattering or ordinary fluorescence corresponds to the $n_{i} 1$ term in the factor $n_{i}\left(n_{f}+1\right)$ that characterizes boson transition rates. Hence the sum over all possible initial and final atom trap states gives a factor of $N$ for the Rayleigh rate. Because all final states are weighted equally, there is no dependence on $\boldsymbol{\delta}$.

Diffraction reflects the particle density. The characteristic $\delta$ of each subprocess is the inverse of the density's characteristic length scale. In a harmonic potential, using natural units, the ground state has size $O(1)$. Thermally occupied states have size $O\left(T^{-1 / 2}\right)$. Hence the most prominent (largest angle) feature of diffraction occurs for $T<T_{c}$ due to scattering off the condensate. The condensate diffraction rate is proportional to $N_{0}^{2}$ for the region $\delta \lesssim 1$ (in the natural oscillator units used throughout). For larger $\delta$, this term drops off with an $\exp \left(-\delta^{2} / 2\right)$ because there just is no more probability to find condensate particles at larger momenta. The relevant $n_{f}$ is indeed $n_{i}-1$. One must also, in principle, add all possible struck atoms coherently. However, the diffraction from the diffuse, thermal cloud corresponding to excited trap states has support only for $\delta \rightarrow 0$ as $N \rightarrow \infty$. Because diffraction is limited in $\delta$, in contrast to the $\delta$-independent Rayleigh scattering, the diffraction contribution to the total cross section has a factor of $\left(\bar{\delta} / k_{i}\right)^{2}$, where $\bar{\delta}$ is the mean momentum transfer for the process and $k_{i}$ is the initial photon momentum.

"Bose-stimulated scattering" is used here to refer to the $n_{i} n_{f}$ term, where coherence among the $n_{f}$ bosons in the final state leads to an enhancement of the rate relative to the totally incoherent Rayleigh rate. This is a dramatic feature of gases of Bose-condensed atoms for $T \lesssim T_{c}$ when several of the lowest-lying states have large occupation numbers. The characteristic $\delta$ is of order the typical thermal particle momentum. This momentum is $O\left(T^{1 / 2}\right)$. However, the sum over $i$ and $f$ does not give cross sections proportional to $N_{0} N_{e}$ or $N_{e}^{2}$, where $N_{e}=N-N_{0}$, even when integrated over all possible $\boldsymbol{\delta}$. Instead, it was shown explicitly that only $O\left(N_{e}^{1 / 3}\right)$ of the $N_{e}$ particles in excited states have an overlap with the ground state after absorption of the photon recoil momentum. The reason that the $n_{i}$ (or $n_{f}$ ) factor does not lead to an $N_{e}$ in the final result is that the typical excited-state particle has an orbit or wave function that is inherently three dimen- 
sional. The $1 / 3$ power comes just from the reduction from the three-dimensional ensemble to the one dimension of contributing, active participants. Transferring a particle from one typical occupied excited state to another with a single impulse from the photon proves to be, if anything, a bit harder. Again, only $O\left(N_{e}^{1 / 3}\right)$ of the $N_{e}$ possible final particles contribute.

The scattering rate from the $0 \leftrightarrow m$ processes grows like $T / \delta^{2}$ for angles corresponding to $\delta^{2} \lesssim 2 T$, and this $T / \delta^{2}$ is the enhancement factor over the Rayleigh differential rate in the corresponding angular region. The angular size of this region grows with $N$, since $T \approx N_{e}^{1 / 3}$. This is in contrast to diffraction from the condensate, which is limited to $\delta \sim O(1)$. The $m \leftrightarrow m^{\prime}$ rate, though monotonically increasing with decreasing $\delta$, is never substantially bigger than the Rayleigh rate. Also, both Bose-stimulated processes fall off exponentially with $\delta^{2}$ for $\delta^{2}>2 T$ again because of the absence of particles with higher momenta.

The heart of the calculation of the Bose-stimulated processes is the estimate of the efficacy of a photon momentum transfer $\boldsymbol{\delta}$ to effect a transition between occupied trap states. The details of the transition matrix elements will depend on the explicit trapping potential. Whatever the potential, the integration over all $\delta$ is two rather than three dimensional because of the constraint of overall energy conservation. This implies that even integrating over all kinematically accessible $\boldsymbol{\delta}$ cannot produce a Bose stimulation factor that is a significant fraction of $N$. Instead, it is generically a fractional power.

If one observes the photon energies with high resolution (instead of integrating over them as done in the calculations presented here), there will be a strong angular dependence to the line shape. In particular, the different processes discussed here produce different photon spectra. At the smallest angles, the dominant diffraction leaves the photon energy unchanged. The Bose-enhanced scattering, dominant at somewhat larger angles, occurs with photon energy shifts of $O(T)$, while Rayleigh scattering has a characteristic, larger shift due to atom recoil.
[1] See, e.g., L. You, M. Lewenstein, and J. Cooper, Phys. Rev. A 50, R3565 (1994); M. Lewenstein, L. You, J. Cooper, and K. Burnett, ibid. 50, 2207 (1994); L. You, M. Lewenstein, R.J. Glauber, and J. Cooper, ibid. 53, 329 (1996), and references therein.

[2] J. Javanainen, Phys. Rev. Lett. 72, 2375 (1993).
[3] H.D. Politzer, Phys. Lett. A 209, 160 (1995).

[4] M.R. Andrews, M.-O. Mewes, N.J. van Druten, D.S. Durfee, D.M. Kurn, and W. Ketterle, Science 273, 84 (1996).

[5] S.A. de Groot, G.J. Hooyman, and S. Sedlam, Proc. R. Soc. London, Ser. A 203, 266 (1950).

[6] H.D. Politzer, Phys. Rev. A (to be published). 